\title{
Correlation between seed size, protein and oil contents, and fatty acid composition in soybean genotypes
}

\author{
By Damián M. Maestri ${ }^{*}$, Diana O. Labuckas ${ }^{a}$, Carlos A. Guzmán ${ }^{a}$ and Laura M. Giorda ${ }^{b}$ \\ ${ }^{a}$ Cátedra de Química Orgánica. Facultad de Ciencias Exactas, Físicas y Naturales, \\ Universidad Nacional de Córdoba. Av. Vélez Sarsfield 299, Córdoba 5000. Argentina. \\ ${ }^{\mathrm{b}}$ Estación Experimental Agropecuaria. INTA Manfredi, Manfredi 5988, Argentina.
}

\section{RESUMEN}

Correlación entre el tamaño de semilla, contenidos en proteínas y aceites y composición en ácidos grasos en genotipos de soja.

Se analizaron 18 genotipos de soja (Glycine $\max$ (L.) Merrill) con grupos de madurez IV, V, VI o VII, cultivados en 1995/96 en la Estación Experimental Agropecuaria (EEA-INTA) de Manfredi y Marcos Juárez, Argentina. El propósito de la investigación fue determinar posibles asociaciones entre el tamaño del grano, los contenidos de proteínas y aceite y la composición de ácidos grasos del mismo. El tamaño del grano varió entre 13.9-21.0 g/100 granos. Los porcentajes de proteínas y aceite estuvieron comprendidos entre $331-448$ y entre $198-267 \mathrm{~g} \mathrm{~kg}^{-1}$, respectivamente, y no mostraron correlaciones significativas con el tamaño del grano. Se observaron correlaciones significativas entre el tamaño del grano y determinados ácidos grasos: positivas con esteárico y oleico y negativa con linoleico. Las asociaciones encontradas podrían ser de utilidad en programas de mejoramiento de soja.

PALABRAS-CLAVE: Aceite - Acido graso - Proteína - Semilla (tamaño) - Soja.

\section{SUMMARY}

Correlation between seed size, protein and oil contents, and fatty acid composition in soybean genotypes.

Eighteen soybean genotypes (Glycine max (L.) Merrill) with maturity groups IV, V, VI or VII were grown in 1995/96 at the Estación Experimental Agropecuaria (EEA-INTA) of Manfredi and Marcos Juárez, Argentina. The aim of this research was to determine possible associations between seed size, protein and oil contents, and fatty composition. Seed size varied between 13.9-21.0 g/100 seeds. Protein and oil contents ranged from 331 to 448 and from 198 to $267 \mathrm{~g} \mathrm{~kg}^{-1}$, respectively, and showed no significant correlation with seed size. There were significant correlations between seed size and individual fatty acids: positive with stearic and oleic and negative with linoleic. The results obtained suggest that seed size and its relationship with individual fatty acids must be considered in soybean breeding programs.

KEY-WORDS: Fatty acid - Oil - Protein - Seed (size) - Soybean.

\section{INTRODUCTION}

The soybean is the most important source of edible vegetable oil and high quality vegetable protein in the world today. Developing soybean varieties with superior chemical composition to meet special food applications has become a high research priority. There are three major factors affecting soybean quality: a) protein and oil content, $b$ ) the chemical components of protein and oil, and c) seed appearance (Liu et al., 1995; Wilson, 1995). Both chemical and physical attributes are definitive factors that help establish grade and price (Sinclair, 1995). During selection of soybeans for a particular food application or a particular seed breeding program, it is important to know whether relationships exist among these quality attributes. Unfortunately, there seems to be little published information on relationships between soybean seed size, protein and oil contents, and fatty acid composition (Liu et al., 1995). This investigation was conducted with 18 soybean cultivars grown in Argentina to determine the nature of these associations.

\section{MATERIALS AND METHODS}

\subsection{Plant material.}

Eighteen soybean genotypes (Glycine max (L.) Merrill) with maturity groups IV, V, VI or VII, were grown in 1995/96. In order to examine the feasibility of environmental variation in chemical composition, the genotypes were grown at the Estación Experimental Agropecuaria (EEA - INTA) from two different localities: Manfredi and Marcos Juarez, Córdoba province, Argentina. Manfredi (latitude $31^{\circ} 49^{\prime}$ S) is 292 meters above mean sea level and had an average monthly temperature of $20.9^{\circ} \mathrm{C}$ during the growing season (november-abril). Marcos Juárez ( $32^{\circ}$ $35 \mathrm{~S}$ ) is 110 meters above mean sea level and had an average monthly temperature of $22.8^{\circ} \mathrm{C}$. Summer rainfall is normally about the same for the two locations; Manfredi received $577.5 \mathrm{~mm}$ and Marcos Juárez had a total of $629.0 \mathrm{~mm}$ during the growing season. The experiment was replicated three times in a split-plot arrangement of a randomized complete block design. Seeds of each lot were harvested separately by hand at maturity when seed moisture was reduced to $10 \%$ or less. 


\subsection{Oil extraction.}

Crude oils were extracted with $n$-hexane in a Soxhlet apparatus for $12 \mathrm{hs}$. After drying the solution with anhydrous sodium sulphate, solvent was removed by vacuum distillation at $30^{\circ} \mathrm{C}$. Oil percentages were determined by weight difference.

\subsection{Fatty acid analysis.}

The lipids were subjected to alkaline saponification (1 $\mathrm{N} \mathrm{KOH}$ in methanol). Unsaponifiable matter was extracted with $n$-hexane. The fatty acid methyl esters (FAME) of total lipids were obtained using $1 \mathrm{~N} \mathrm{H}_{2} \mathrm{SO}_{4}$ in methanol and analyzed by gas chromatography (GC) according to Maestri and Guzmán (1993). The theoretical iodine number was calculated according to Carreras et al. (1989).

\subsection{Protein content.}

The nitrogen content was determined by the Kjeldahl method and it was converted to protein content by using the conversion factor 6.25 (AOAC, 1980).

\subsection{Statistical analyses.}

All chemical and physical determinations were conducted in triplicate. Statistical differences among cultivars were estimated from ANOVA test at the $5 \%$ level $(P=0.05)$ of significance for all parameters evaluated. Whenever ANOVA indicated significant difference, a pairwise comparison of mean by Least Significant Difference test (LSD) was carried out. Correlation analysis was performed employing Pearson's test. Correlation coefficients were calculated on cultivar values for each location.

\section{RESULTS AND DISCUSSION}

Seed size presented a statistically significant variation among cultivars, ranging from 13.9 to 20.7 g/100 seeds; cultivars of maturity group IV showed the highest values (Tables I and II).

Statistically significant differences were found among genotypes for protein and oil contents. Protein content ranged from 331 to $448 \mathrm{~g} \mathrm{~kg}^{-1}$, whereas oil content constituted more than $198 \mathrm{~g} \mathrm{~kg}^{-1}$ in all cultivars. Protein and oil contents varied significantly between locations; cultivars grown at Manfredi showed the highest values (Tables I and II). There was no a negative correlation between protein and oil contents as indicated previously (Leffel and Rhodes, 1993; Liu et al., 1995).

Table 1

Seed size ( $\mathrm{g} / 100$ seeds), protein and oil contents $\left(\mathrm{g} \mathrm{kg}^{-1}\right)$, fatty acid composition (\% of total fatty acids), percentage of unsaturated (\% US), oleic to linolenic (O/Ln) ratios and iodine values (IVs) of seed oils from 18 soybean cultivars grown at Manfredi. Means $(n=3)$ from each cultivar followed by the same letter within each column are not significantly different at $P<0.05$ by LSD pairwise comparison of means

\begin{tabular}{|c|c|c|c|c|c|c|c|c|c|c|c|c|c|c|}
\hline & Seed size & Protein & Oil & $16: 0$ & 18:0 & 18:1 & $18: 2$ & $18: 3$ & $20: 0$ & $20: 1$ & 22:0 & \%US & Olnn & IVs \\
\hline \multicolumn{15}{|l|}{ Maturity group IV } \\
\hline Don Mario 49 & $20.3^{\mathrm{a}}$ & $412^{g h}$ & $254^{\text {bc }}$ & $11.3^{\text {odel }}$ & $4.2^{\text {cde }}$ & $27.4^{\mathrm{a}}$ & $50.2^{j}$ & $6.8^{\prime}$ & $\operatorname{tr}^{*}$ & $\operatorname{tr}$ & $\operatorname{tr}$ & $84.4^{\mathrm{abc}}$ & $4.0^{\mathrm{a}}$ & $134.4^{\mathrm{jk}}$ \\
\hline Dekalb 458 & $19.6^{\mathrm{a}}$ & $448^{\mathrm{a}}$ & $243^{d}$ & $10.0^{\| 1}$ & $4.5^{\mathrm{bc}}$ & $25.2^{\mathrm{b}}$ & $51.3^{\mathrm{ij}}$ & $7.3^{\mathrm{hi}}$ & 0.6 & 0.4 & 0.4 & $84.3^{\mathrm{abc}}$ & $3.4^{\mathrm{b}}$ & $136.2^{\mathrm{j}}$ \\
\hline Ofpec Bonaerense & $20.7^{\mathrm{a}}$ & $418^{\lg }$ & $238^{\mathrm{det}}$ & $9.9^{\prime}$ & $4.9^{\mathrm{ab}}$ & $23.8^{\mathrm{c}}$ & $52.2^{\mathrm{hi}}$ & $7.7^{g^{h}}$ & 0.7 & 0.4 & 0.3 & $84.1^{\text {bcd }}$ & $3.1^{\mathrm{bc}}$ & $137.5^{i}$ \\
\hline Dorada 48 & $20.6^{\mathrm{a}}$ & $430^{\text {cdet }}$ & $248^{\text {bcd }}$ & $10.6^{\text {lghij }}$ & $5.1^{\mathrm{a}}$ & $24.9^{b}$ & $50.6^{j}$ & $7.1^{\prime}$ & 0.4 & 0.4 & 0.4 & $83.0^{\prime}$ & $3.5^{\mathrm{b}}$ & $134.0^{k}$ \\
\hline \multicolumn{15}{|l|}{ Maturity Group V } \\
\hline Rojas INTA & $17.4^{\mathrm{b}}$ & $436^{\mathrm{abcd}}$ & $267^{\mathrm{a}}$ & $11.4^{\text {cde }}$ & $3.8^{\prime g}$ & $22.2^{d}$ & $53.5^{\text {tg }}$ & $7.7^{\text {gh }}$ & 0.6 & 0.3 & 0.4 & $83.8^{\mathrm{bod}}$ & $2.9^{\alpha d}$ & $138.5^{\text {hi }}$ \\
\hline Asgrow 5153 & $16.2^{\mathrm{bcd}}$ & $439^{\mathrm{abc}}$ & $232^{\text {ef }}$ & $11.5^{\mathrm{cd}}$ & $3.7^{19}$ & $18.6^{\prime}$ & $54.6^{\text {bcdef }}$ & $10.0^{\mathrm{a}}$ & 0.6 & 0.4 & 0.4 & $83.6^{\text {cde }}$ & $1.9^{\mathrm{hi}}$ & $143.4^{\mathrm{cd}}$ \\
\hline Reyna 52 & $15.9^{\text {cde }}$ & $423^{\mathrm{efg}}$ & $217^{9}$ & $10.4^{g h \|}$ & $3.9^{\text {def }}$ & $20.5^{\lg }$ & $55.5^{\mathrm{bc}}$ & $8.3^{\text {el }}$ & 0.6 & 0.3 & 0.4 & $84.6^{\mathrm{ab}}$ & $2.5^{\mathrm{et}}$ & $142.2^{\text {cde }}$ \\
\hline Batara 54 & $13.9^{\prime}$ & $424^{\text {detg }}$ & $241^{d \theta}$ & $10.7^{\text {elghi }}$ & $3.5^{\mathrm{gh}}$ & $20.1^{\text {gh }}$ & $55.1^{\text {bod }}$ & $9.1^{\circ}$ & 0.6 & 0.5 & 0.4 & $84.7^{\mathrm{ab}}$ & $2.2^{\text {lgh }}$ & $143.3^{\text {cd }}$ \\
\hline Asgrow 5780 & $16.2^{\mathrm{bcd}}$ & $396^{\prime}$ & $229^{\prime}$ & $11.3^{\text {cdet }}$ & $4.0^{\text {def }}$ & $19.9^{g h}$ & $55.4^{\mathrm{bc}}$ & $7.8^{9}$ & 0.6 & 0.4 & 0.4 & $83.5^{\text {cde }}$ & $2.5^{\mathrm{det}}$ & $140.1^{1 g}$ \\
\hline Torcacita 58 & $15.7^{\text {ode }}$ & $411^{g h}$ & $198^{h}$ & $10.8^{\text {detgh }}$ & $3.9^{\text {det }}$ & $20.9^{\text {ef }}$ & $54.5^{\text {cdef }}$ & $7.8^{1 g}$ & 0.7 & 0.5 & 0.4 & $83.7^{\text {cde }}$ & $2.7^{\mathrm{de}}$ & $139.4^{g h}$ \\
\hline Conesa INTA & $16.8^{\mathrm{bc}}$ & $427^{\text {cdef }}$ & $258^{\mathrm{ab}}$ & $11.4^{\text {ode }}$ & $4.2^{\text {cd }}$ & $21.5^{\mathrm{de}}$ & $53.1^{g h}$ & $8.5^{\mathrm{de}}$ & 0.4 & 0.3 & 0.4 & $83.3^{d e t}$ & $2.5^{\mathrm{del}}$ & $139.1^{\text {gh }}$ \\
\hline Don Mario 57 & $14.4^{\prime}$ & $432^{\text {bode }}$ & $245^{\alpha d}$ & $11.8^{\mathrm{bc}}$ & $3.8^{\text {etg }}$ & $21.0^{\theta t}$ & $53.6^{\text {elg }}$ & $8.3^{\text {ot }}$ & 0.6 & 0.3 & 0.5 & $83.2^{\mathrm{ef}}$ & $2.5^{\mathrm{el}}$ & $139.1^{\text {gh }}$ \\
\hline \multicolumn{15}{|l|}{ Maturity Group VI } \\
\hline Golondrina 65 & $16.9^{\mathrm{bc}}$ & $377^{1}$ & $240^{d \theta}$ & $11.3^{\text {cde }}$ & $3.5^{\text {gh }}$ & $20.3^{\text {tgh }}$ & $55.0^{\text {bod }}$ & $8.6^{\mathrm{de}}$ & 0.5 & 0.3 & 0.4 & $84.2^{\mathrm{bcd}}$ & $2.3^{\operatorname{lgh}}$ & $142.0^{d e}$ \\
\hline NK 642 & $15.0^{\text {def }}$ & $420^{\text {efg }}$ & $231^{\text {el }}$ & $10.3^{\text {hij }}$ & $3.6^{19}$ & $21.0^{\text {el }}$ & $54.7^{\text {bode }}$ & $9.2^{\mathrm{bc}}$ & 0.4 & 0.3 & 0.3 & $85.2^{\mathrm{a}}$ & $2.3^{\text {el }}$ & $143.6^{\mathrm{abc}}$ \\
\hline Asgrow 6443 & $14.9^{\text {ef }}$ & $402^{h i}$ & $239^{d e t}$ & $11.0^{\text {detgh }}$ & $3.3^{h}$ & $19.6^{h}$ & $57.0^{\mathrm{a}}$ & $8.4^{\mathrm{\theta}}$ & 0.6 & $\operatorname{tr}$ & $\operatorname{tr}$ & $85.2^{\mathrm{a}}$ & $2.3^{\operatorname{lgh}}$ & $144.1^{\mathrm{ab}}$ \\
\hline \multicolumn{15}{|l|}{ Maturity Group VII } \\
\hline Asgrow 7306 & $16.6^{\mathrm{bc}}$ & $402^{\mathrm{hj}}$ & $203^{h}$ & $11.1^{\text {odelg }}$ & $4.0^{\mathrm{det}}$ & $20.6^{l g}$ & $54.0^{\text {delg }}$ & $8.9^{\mathrm{cd}}$ & 0.5 & 0.3 & 0.5 & $83.8^{\mathrm{bcd}}$ & $2.3^{l g}$ & $141.2^{\text {et }}$ \\
\hline Asgrow 7894 & $19.4^{\mathrm{a}}$ & $444^{\mathrm{ab}}$ & $217^{9}$ & $12.7^{\mathrm{a}}$ & $3.8^{\text {etg }}$ & $18.3^{\prime}$ & $55.5^{\mathrm{bc}}$ & $9.7^{\mathrm{ab}}$ & $\operatorname{tr}$ & $\operatorname{tr}$ & $\operatorname{tr}$ & $83.5^{\text {ode }}$ & $1.9^{\mathrm{hi}}$ & $143.6^{\mathrm{abc}}$ \\
\hline Asgrow 7986 & $17.2^{\mathrm{b}}$ & $429^{\text {cdef }}$ & $254^{b c}$ & $12.4^{\mathrm{ab}}$ & $3.2^{h}$ & $18.4^{\prime}$ & $55.8^{\mathrm{b}}$ & $10.0^{\mathrm{a}}$ & $\operatorname{tr}$ & $\operatorname{tr}$ & $\operatorname{tr}$ & $84.2^{\mathrm{bcd}}$ & $1.8^{i}$ & $145.1^{\mathrm{a}}$ \\
\hline
\end{tabular}

$*$ tr, trace $<0.1 \%$. 
Table II

Seed size ( $\mathrm{g} / 100$ seeds), protein and oil contents $\left(\mathrm{g} \mathrm{kg}^{-1}\right)$, fatty acid composition (\% of total fatty acids), percentage of unsaturated (\% US), oleic to linolenic (O/Ln) ratios and iodine values (IVs) of seed oils from 18 soybean cultivars grown at Marcos Juárez. Means $(n=3)$ from each cultivar followed by the same letter within each column are not significantly different at $P<0.05$ by LSD pairwise comparison of means

\begin{tabular}{|c|c|c|c|c|c|c|c|c|c|c|c|c|c|c|}
\hline & Seed size & Protein & Oil & $16: 0$ & $18: 0$ & $18: 1$ & $18: 2$ & $18: 3$ & $20: 0$ & $20: 1$ & $22: 0$ & \%US & O/Ln & IVs \\
\hline \multicolumn{15}{|l|}{ Maturity group IV } \\
\hline Don Mario 49 & $19.9^{\mathrm{a}}$ & $359^{j}$ & $206^{i}$ & $11.5^{\text {ode }}$ & $4.5^{\mathrm{ab}}$ & $23.5^{\mathrm{a}}$ & $52.2^{i}$ & $7.2^{\mathrm{c}}$ & 0.3 & 0.2 & 0.3 & $83.1^{\mathrm{g}}$ & $3.2^{\mathrm{a}}$ & $135.9^{h}$ \\
\hline Dekalb 458 & $19.3^{\mathrm{ab}}$ & $412^{\mathrm{a}}$ & $211^{9}$ & $10.7^{\mathrm{efg} g}$ & $4.5^{\mathrm{ab}}$ & $23.1^{\mathrm{a}}$ & $53.9^{g h}$ & $7.5^{\mathrm{de}}$ & 0.1 & 0.1 & $\operatorname{tr}^{*}$ & $84.6^{\mathrm{abc}}$ & $3.1^{\mathrm{ab}}$ & $139.0^{f g}$ \\
\hline Ofpec Bonaerense & $20.5^{\mathrm{a}}$ & $381^{\prime}$ & $215^{t}$ & $10.1^{g}$ & $4.6^{\mathrm{a}}$ & $22.3^{a b}$ & $54.7^{\mathrm{tgh}}$ & $7.9^{\text {cde }}$ & 0.2 & 0.1 & 0.2 & $85.0^{\mathrm{ab}}$ & $2.8^{\mathrm{abc}}$ & $141.0^{\text {ef }}$ \\
\hline Dorada 48 & $21.0^{\mathrm{a}}$ & $363^{i}$ & $211^{9}$ & $11.3^{\text {ode }}$ & $4.9^{\mathrm{abc}}$ & $22.5^{\mathrm{ab}}$ & $53.5^{\mathrm{hi}}$ & $7.4^{\mathrm{de}}$ & 0.2 & $\operatorname{tr}$ & $\operatorname{tr}$ & $83.4^{\mathrm{efg}}$ & $3.1^{\mathrm{ab}}$ & $137.6^{g h}$ \\
\hline \multicolumn{15}{|l|}{ Maturity Group V } \\
\hline Rojas INTA & $17.7^{\mathrm{bc}}$ & $377^{9}$ & $218^{\theta}$ & $11.2^{\text {odet }}$ & $3.7^{\text {bedet }}$ & $20.6^{\mathrm{cd}}$ & $55.6^{\text {defg }}$ & $7.9^{\text {cde }}$ & 0.4 & $\operatorname{tr}$ & 0.4 & $84.1^{\mathrm{bcd}}$ & $2.6^{\mathrm{abod}}$ & $141.0^{\text {ef }}$ \\
\hline Asgrow 5153 & $16.4^{\text {de }}$ & $392^{c}$ & $214^{t}$ & $11.7^{\mathrm{bcd}}$ & $3.9^{\mathrm{ab} o d \mathrm{e}}$ & $19.1^{\text {def }}$ & $54.5^{\mathrm{tgh}}$ & $9.4^{\mathrm{a}}$ & 0.7 & 0.2 & 0.5 & $83.2^{g}$ & $2.0^{\mathrm{et}}$ & $142.0^{\text {cdet }}$ \\
\hline Reyna 52 & $16.1^{\text {odet }}$ & $388^{\mathrm{de}}$ & $222^{d}$ & $11.8^{\mathrm{abcd}}$ & $4.1^{\mathrm{abode}}$ & $19.3^{\mathrm{det}}$ & $56.7^{\text {cde }}$ & $7.6^{\mathrm{de}}$ & 0.2 & $\operatorname{tr}$ & 0.2 & $83.6^{\mathrm{def}}$ & $2.5^{\mathrm{bcde}}$ & $141.1^{\mathrm{et}}$ \\
\hline Batara 54 & $14.0^{9}$ & $348^{\prime}$ & $215^{t}$ & $11.0^{\mathrm{det}}$ & $3.2^{\text {ef }}$ & $17.4^{9}$ & $57.1^{\text {bod }}$ & $9.3^{\mathrm{a}}$ & 0.2 & $\operatorname{tr}$ & 0.2 & $83.8^{\text {ode }}$ & $1.9^{f}$ & $146.6^{\mathrm{a}}$ \\
\hline Asgrow 5780 & $16.0^{\text {odef }}$ & $351^{k}$ & $214^{t}$ & $11.1^{\mathrm{def}}$ & $4.3^{\mathrm{abod}}$ & $20.5^{\circ d}$ & $54.1^{\text {gh }}$ & $8.9^{\mathrm{ab}}$ & 0.7 & 0.1 & 0.1 & $83.7^{\text {cde }}$ & $2.3^{\text {cdel }}$ & $141.2^{\mathrm{ef}}$ \\
\hline Torcacita 58 & $15.2^{\text {defg }}$ & $342^{m}$ & $235^{\mathrm{a}}$ & $11.1^{\text {det }}$ & $3.3^{\text {ef }}$ & $18.4^{\mathrm{efg}}$ & $58.9^{\mathrm{a}}$ & $7.9^{\text {cd }}$ & 0.2 & $\operatorname{tr}$ & 0.2 & $85.2^{\mathrm{a}}$ & $2.3^{\text {odef }}$ & $145.0^{\mathrm{abc}}$ \\
\hline Conesa INTA & $16.1^{\text {ddel }}$ & $351^{k}$ & $209^{h}$ & $11.3^{\text {cde }}$ & $3.6^{\text {odet }}$ & $17.8^{l g}$ & $57.7^{\mathrm{abc}}$ & $9.0^{\mathrm{ab}}$ & 0.2 & 0.2 & 0.2 & $84.7^{\mathrm{abc}}$ & $2.0^{e f}$ & $145.6^{\mathrm{ab}}$ \\
\hline Don Mario 57 & $14.5^{\mathrm{tg}}$ & $359^{\prime}$ & $217^{\circ}$ & $11.7^{\mathrm{bod}}$ & $3.5^{\mathrm{del}}$ & $18.5^{\text {efg }}$ & $56.9^{\text {bod }}$ & $8.9^{\mathrm{ab}}$ & 0.2 & $\operatorname{tr}$ & 0.2 & $84.3^{\mathrm{abc}}$ & $2.1^{\text {det }}$ & $144.3^{\mathrm{abcd}}$ \\
\hline \multicolumn{15}{|l|}{ Maturity Group VI } \\
\hline Golondrina 65 & $16.5^{\text {od }}$ & $365^{i}$ & $227^{\mathrm{c}}$ & $12.0^{\mathrm{abc}}$ & $3.9^{\mathrm{abode}}$ & $20.2^{\text {od }}$ & $55.9^{\text {det }}$ & $7.4^{\mathrm{de}}$ & 0.2 & 0.1 & 0.2 & $83.7^{\text {ode }}$ & $2.7^{\mathrm{abod}}$ & $140.1^{\text {elg }}$ \\
\hline NK 642 & $15.1^{\text {delg }}$ & $331^{n}$ & $233^{b}$ & $10.4^{\mathrm{fg}}$ & $3.3^{\text {ef }}$ & $19.5^{\text {dde }}$ & $56.2^{\text {cdel }}$ & $8.5^{\mathrm{bc}}$ & 0.3 & 0.2 & 0.4 & $84.4^{\mathrm{abc}}$ & $2.3^{\text {odef }}$ & $143.3^{\text {bode }}$ \\
\hline Asgrow 6443 & $14.7^{\mathrm{efg}}$ & $384^{\theta}$ & $203^{j}$ & $11.2^{\text {cdef }}$ & $3.6^{\text {odet }}$ & $16.9^{\text {efg }}$ & $58.6^{\mathrm{ab}}$ & $8.7^{\mathrm{ab}}$ & 0.3 & 0.2 & 0.3 & $84.4^{\mathrm{abc}}$ & $1.9^{\prime}$ & $145.6^{\mathrm{ab}}$ \\
\hline \multicolumn{15}{|l|}{ Maturity Group VII } \\
\hline Asgrow 7306 & $16.9^{\circ}$ & $373^{h}$ & $199^{k}$ & $11.3^{\text {cde }}$ & $4.0^{\mathrm{abcde}}$ & $21.1^{\mathrm{bc}}$ & $54.7^{\text {ghh }}$ & $8.5^{\mathrm{bc}}$ & 0.3 & tr & $\operatorname{tr}$ & $84.3^{\mathrm{abc}}$ & $2.5^{\mathrm{abod}}$ & $141.5^{\mathrm{det}}$ \\
\hline Asgrow 7894 & $19.3^{\mathrm{ab}}$ & $398^{\mathrm{b}}$ & $209^{h}$ & $12.4^{\mathrm{ab}}$ & $3.9^{\mathrm{abcde}}$ & $19.0^{\text {detg }}$ & $55.0^{\text {etgh }}$ & $9.1^{\mathrm{ab}}$ & 0.4 & $\operatorname{tr}$ & 0.1 & $83.1^{9}$ & $2.1^{\text {odef }}$ & $141.8^{\text {del }}$ \\
\hline Asgrow 7986 & $17.1^{\circ}$ & $387^{d}$ & $233^{b}$ & $12.6^{\mathrm{a}}$ & $3.5^{\mathrm{det}}$ & $19.2^{\text {def }}$ & $55.1^{\text {elgh }}$ & $9.2^{\mathrm{ab}}$ & 0.3 & $\operatorname{tr}$ & 0.1 & $83.4^{\mathrm{fg}}$ & $2.1^{\text {et }}$ & $142.3^{\text {cde }}$ \\
\hline
\end{tabular}

${ }^{*} \operatorname{tr}$, trace $<0.1 \%$

The fatty acid compositions were characterized by a high proportion of unsaturated fatty acids which varied between 83.0 and $85.2 \%$. The relative percentage of major fatty acids ranged from 9.9 to 12.7 for palmitic acid (16:0), 3.2 to 5.1 for stearic acid (18:0), 16.9 to 27.4 for oleic acid (18:1), 50.2 to 58.9 for linoleic acid (18:2) and 6.8 to 10.0 for linolenic acid (18:3). Genotypes of maturity group IV from two localities studied, presented the highest percentages of oleic acid and the lowest percentages of linoleic and linolenic acids. As a consequence, the highest $\mathrm{O} / \mathrm{Ln}$ ratios and the smallest IVs were found for genotypes of maturity group IV.

Seed size showed no significant correlation with both protein and oil contents. Similarly, there was no significant correlation between total percentage of unsaturated fatty acids and seed size. However, when individual fatty acids were considered, it was found a significant correlation $(P<0.05)$ with seed size: positive with stearic and oleic acids, and negative with linoleic acid. In addition, there was a negative correlation between seed size and linolenic acid percentage but it was not significant at $P<0.05$ level (Tables III and IV). These findings are in general agreement with those previously published by Liu et al., (1995).
Table III

Correlation coeficients between chemical and physical parameters analyzed from 18 soybean cultivars grown at Manfredi

\begin{tabular}{lcccccc}
\hline \multicolumn{1}{c}{ Factors } & $18: 0$ & $18: 1$ & $18: 2$ & $18: 3$ & Oil & Seed size \\
\hline $18: 1$ & $0.741^{\mathrm{a}}$ & & & & & \\
$18: 2$ & $-0.774^{\mathrm{a}}$ & $-0.894^{\mathrm{a}}$ & & & & \\
$18: 3$ & $-0.656^{\mathrm{a}}$ & $-0.846^{\mathrm{a}}$ & $0.638^{\mathrm{a}}$ & & & \\
Seed size & $0.703^{\mathrm{a}}$ & $0.592^{\mathrm{a}}$ & $-0.587^{\mathrm{a}}$ & -0.383 & 0.226 & \\
Protein & & & & & 0.451 & 0.209 \\
\hline
\end{tabular}

a These numbers correspond correlations wich are significant at $P<0.05$.

Table IV

Correlation coeficients between chemical and physical parameters analyzed from 18 soybean cultivars grown at Marcos Juárez

\begin{tabular}{lcccccc}
\hline \multicolumn{1}{c}{ Factors } & $18: 0$ & $18: 1$ & $18: 2$ & $18: 3$ & Oil & Seed size \\
\hline $18: 1$ & $0.702^{\mathrm{a}}$ & & & & & \\
$18: 2$ & $-0.721^{\mathrm{a}}$ & $-0.901^{\mathrm{a}}$ & & & & \\
$18: 3$ & $-0.536^{\mathrm{a}}$ & $-0.830^{\mathrm{a}}$ & $0.654^{\mathrm{a}}$ & & & \\
Seed size & $0.631^{\mathrm{a}}$ & $0.541^{\mathrm{a}}$ & $-0.611^{\mathrm{a}}$ & -0.402 & 0.295 & \\
Protein & & & & & 0.328 & 0.267 \\
\hline
\end{tabular}

a These numbers correspond correlations which are significant at $P<0.05$. 
Regarding the relationships among the major individual unsaturated fatty acids, there was a negative correlation between oleic acid with each of two polyunsaturated acids (18:2 and 18:3), while a positive correlation between linoleic and linolenic acids was observed at $\mathrm{P}<0.05$ level. These results agree with those obtained by Burton et al. (1983) and Liu et al. (1995). Also, the following pairs of relationships were found: positive between 18:0-18:1, and negative between 18:0-18:2 and 18:0-18:3.

Current biochemical evidence indicates that in soybeans and in several other plant species the polyunsaturated fatty acids (18:2 and 18:3) are produced by the consecutive desaturation of 18:1 (Wilson et al., 1980). Liu et al. (1995) have suggested that genes encoding enzymes responsible for desaturation of oleoyl-phosphatidylcholine (PC) to linoleoyl-PC and further to linolenoyl-PC are related not only to each other but also to genes controlling seed size.

In summary, the results obtained in this work suggest that, besides oil and protein contents and fatty acid composition, seed size and its relationship with individual fatty acids and the association among these fatty acids, must be considered in soybean breeding programs.

\section{ACKNOWLEDGEMENTS}

Cooperative investigations between Cátedra de Química Orgánica (FCEF y N - UNC) and EEA - INTA Manfredi, Córdoba. This research was supported by grants from CONICET and CONICOR.

\section{REFERENCES}

AOAC (1980).- «Official Methods of Analysis of the Association of Official Analytical Chemists".- Horwitz, W. (Ed.). Washingtong D.C., USA.

Burton, J.W., Wilson, R.F. and Brim, C.A. (1983).«Recurrent selection in soybeans. IV. Selection for increased oleic acid percentage in seed oil».-Crop Science 23: 744-747.

Carreras, M.E., Fuentes, E. and Guzmán, C.A. (1989)."Chemotaxonomy of seed lipids of Cucurbitaceae grown in Argentina».-Biochem. Syst. Ecol. 17 (4): 287-291.

Leffel, R.C. and Rhodes, W.K. (1993).- «Agronomic performance and economic value of high-seed-protein soybean"--J. Prod. Agric. 6: 365-368.

Liu, K., Orthoefer, F. and Brown, E.A. (1995)."Association of seed size with genotypic variation in the chemical constituents of soybeans".-J. Am. Oil Chem. Soc. 72: 189-192.

Maestri, D.M. and Guzmán, C.A. (1993).-«Chemical composition of tobacco seeds (Nicotiana tabacum L.) from Argentina».-J. Sci. Food Agric. 61: 227-230.

Sinclair, J.B. (1995).- - «Reevaluation of grading standards and discounts for fungus-damaged soybeans seeds".-J. Am. Oil Chem. Soc. 72: 1415-1419.

Wilson, R.F., Weissinger, H.H., Buck, J.A. and Faulkner, G.D. (1980)._ «Involvement of phospholipids in polyunsaturated fatty acid synthesis in developing soybean cotyledons".-Plant Physiol. 66: 545-549.

Wilson, R.F. (1995).- «Dealing with the problems of fungal damage in soybean and other oilseeds".-J. Am. Oil Chem. Soc. 72: 1413-1414. 